\title{
Experimental Investigation of Hot Carriers in Terahertz Quantum Cascade Lasers
}

\author{
G. Scamarcio $^{a, *}$, M.S. Vitiello ${ }^{a}$ And V. Spagnolo ${ }^{b}$ \\ ${ }^{a}$ INFM-CNR Regional Laboratory LIT3, Physics Department \\ University of Bari, via Amendola 173, 70126 Bari, Italy \\ ${ }^{b}$ INFM-CNR Regional Laboratory LIT3, Physics Department \\ Politecnico di Bari, Italy
}

\begin{abstract}
The nature of the electron distribution and the electron-lattice energy relaxation phenomena in all classes of quantum cascade lasers operating in the $\mathrm{THz}$ range, namely, resonant-phonon, bound-to-continuum, and interlaced photon-phonon designs are reviewed. Thermalized hot-electron distributions are found in all cases. However, electronic temperatures of individual conduction subband are strongly influenced by the specific quantum design and the actual electron-lattice energy relaxation channels. A wealth of information was obtained both below and above laser threshold from the analysis of micro-probe band-to-band photoluminescence spectra recorded with a spatial resolution of $\approx 2 \mu \mathrm{m}$. The influence of the detailed knowledge of the hot electron distributions on the design of improved $\mathrm{THz}$ quantum cascade lasers aiming at high temperature operation will be discussed.
\end{abstract}

PACS numbers: 42.55.Px, 68.65.Fg

\section{Introduction}

$\mathrm{THz}$ quantum cascade lasers (QCLs) are semiconductor based sources of coherent radiation covering the frequency range between 1.6 and $5 \mathrm{THz}$ $(60-188 \mu \mathrm{m})[1,2]$. The electronic distribution in the latter class of devices is expected to be significantly different with respect to QCLs working in the midinfrared. While in the latter case the electron-optical phonon interaction is the main electronic energy relaxation channel, in THz QCLs additional processes, such as inter- and intra-subband electron-electron (e-e), electron-impurity, and interface roughness scattering lead to the establishment of a non-equilibrium hot electron distribution. In addition, thermally induced leakage of electrons into delocalized continuum-like states competes with the injection into the upper laser level and thus may contribute to the formation of hot electrons. At electronic sheet

*corresponding author; e-mail: scamarcio@fisica.uniba.it 
densities $\geq 10^{11} \mathrm{~cm}^{-2}$ the e-e scattering is fast enough to create Boltzmann-like subband distributions characterized by electronic temperatures $T_{\mathrm{e}}^{j}$ higher than the lattice one $\left(T_{\mathrm{L}}\right)$ at injected currents close to the laser threshold. Although subband thermalization and the related concept of subband temperature is questionable at densities $<10^{9} \mathrm{~cm}^{-2}$, but it still holds at the densities used in THz QCLs (3-5 $\left.\times 10^{10} \mathrm{~cm}^{-2}\right)[3]$.

In this paper, we extract and compare the electronic temperatures and the electron-lattice coupling of THz QCLs based on resonant-phonon with one well injector (RP-SW) and the bound-to-continuum (BTC) active region schemes. The employed technique is based on micro-probe band-to-band photoluminescence steady-state measurements in which electrons and holes have the same temperature and the power given to the carrier system by the external electrical excitation must be balanced by the power lost from the carrier system to the lattice by carrier-phonon interactions. The temperature of the carrier system increases until such a balance is reached.

\section{Investigated structures}

GaAs $/ \mathrm{Al}_{0.15} \mathrm{Ga}_{0.85} \mathrm{As}$ QCLs based on a RP-SW and a BTC gain medium designs have been studied. The resonant-phonon device, presented in this work, has been designed for emission at $1.9 \mathrm{THz}$ (sample (a)). The quantum design is a combination of resonant tunneling and direct electron-LO-phonon scattering [4]. In particular, the investigated device exploits a new design characterized by a single well injector. The structure was fabricated into a double-metal waveguide since it offers near unity mode confinement and lower losses at low frequencies. The fabrication was done using copper-to-copper thermo-compression wafer bonding, back side substrate removal, and dry etching to form the ridges as described in Ref. [4]. A laser bar $80 \mu \mathrm{m}$ wide and $0.47 \mathrm{~mm}$ long has been investigated in the present work. The BTC QCL (sample (b)) is based on an active region scheme, that uses a miniband $\left(M_{1}\right)$ for the depopulation of the lower radiative state via intra-miniband e-e scattering and whose width is higher than the photon energy. The active region consists of 90 periods and is sandwiched between upper $(80 \mathrm{~nm}$ thick) and lower (600 nm thick) GaAs layers which are doped at levels of $n=$ $5 \times 10^{18} \mathrm{~cm}^{-3}$ and $n=2 \times 10^{18} \mathrm{~cm}^{-3}$, respectively. This allows the realization of a surface-plasmon optical waveguide [1]. The devices have been wet-etched into ridge cavities $150 \mu \mathrm{m}$ wide and $12 \mu \mathrm{m}$ deep. Standard photolithographic procedures allowed the realization of laser bars $2 \mathrm{~mm}$ long showing laser emission at $2.83 \mathrm{THz}[5,6]$.

\section{Experimental technique}

The experimental method is based on the microprobe band-to-band photoluminescence (PL) that proved successful for the investigation of mid-IR and $\mathrm{THz}$ QCLs $[7,8]$. The photoluminescence signals were obtained by focusing a selected 
line of the $\mathrm{Kr}^{+}$laser, working in CW mode, directly onto the QCL front facet down to a spot of about $2 \mu \mathrm{m}$ diameter by using an $80 \times$ long-working-distance $(5 \mathrm{~mm})$ microscope objective lens with numerical aperture $\approx 0.65$. The laser spot is visualized by means of a camera directly connected to a personal computer. The $\mathrm{Kr}^{+}$laser plasma lines were blocked by means of a prism monochromator filter having a spectral range $400-800 \mathrm{~nm}$, a band pass $<10 \mathrm{~nm}$ and a transmission $\approx 0.75$. The devices were mounted into a helium-flow micro-cryostat wired with micro-coaxial cables and kept at a fixed heat sink temperature controlled with a calibrated Si-diode mounted close to the device. The sample position was controlled by a two-dimensional piezoelectric translation stage with $0.1 \mu \mathrm{m}$ spatial resolution. Real-time multiple acquisitions, synchronized with the $x-y$ piezoelectric translation stage, allowed the complete mapping of samples. The PL signal was dispersed using a $0.64 \mathrm{~m}$ monochromator equipped with a single $110 \times 110 \mathrm{~mm}^{2}$ grating of 600 lines/mm and detected with a Si charge coupled device (CCD) cooled to $140 \mathrm{~K}$ so that the dark current level remains lower than 1 electron/pixel/hour. Laser-induced heating of the samples was avoided by keeping the incident power density at values $\approx 200-300 \mathrm{~W} / \mathrm{cm}^{2}$. Under these experimental conditions the density of photogenerated carriers is $0.7-1.85 \times 10^{15} \mathrm{~cm}^{-3}$, i.e. lower than the active region main doping. This allows maintaining the electronic distribution practically unperturbed thus providing only valence band holes needed to probe the electronic population via band-to-band radiative recombination.

\section{Results and discussion}

Figures 1 and 2 shows the results of the PL experiments performed on sample (a). The PL spectrum of Fig. 1 has been collected when an electrical power $\mathrm{P}=$ $0.6 \mathrm{~W}$ is dissipated in the device. The two main peaks correspond to band-to-band transitions $1 \rightarrow k$ between the injector ground state $j=1$, and two distinctive valence subbands $k$, as marked on the conduction and valence band structures of Figs. $2 \mathrm{~b}$ and $\mathrm{c}$, respectively. The structure on the high energy tail of the main peaks is due to the transitions $4 \rightarrow k$ between the upper laser level $j=4$ and the valence subbands. This level becomes progressively populated as an effect of the injected current.

To extract the local lattice temperature we used the red shift of the main PL peak as a function of $P$ as a thermometric property, providing that the voltage induced shift of the confinement energies is properly considered. The local lattice temperature of the active region has been obtained by comparing the energy shift of the main PL band $\Delta E_{\mathrm{p}}$ with a calibration curve, collected by probing the device at zero current while varying the heat sink temperature, following the experimental method described in Refs. [7] and [8]. The procedure employed for the determination of the electronic temperatures of each subband $\left(T_{\mathrm{e}}^{j}\right)$ is reported in Fig. 1. The $T_{\mathrm{e}}^{j}$ values have been extracted from the line shape analysis of photoluminescence spectra. The analysis of the PL line shape is based on the following 


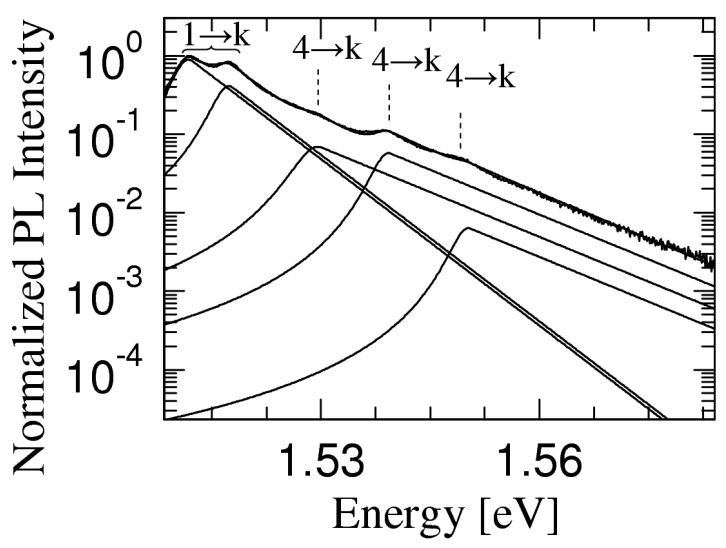

Fig. 1. Dashed line: photoluminescence spectrum of sample (a) measured at $P=$ $0.6 \mathrm{~W}$. Solid line: calculated PL components peaked at the theoretical energies of relevant $j \rightarrow k$ transition. The low energy side of each curve is a Lorentzian with $\mathrm{HWHM}=3.2 \mathrm{meV}$. The high energy side is an exponential decay function $\propto \exp \left(-\left(E-E_{j \rightarrow k}\right) / k_{\mathrm{B}} T_{\mathrm{e}}^{j}\right)$.

expression:

$$
I_{\mathrm{PL}}(E) \propto \sum_{j=1}^{m} \sum_{k=1}^{n} A_{j k} E_{j k}^{4}\left|\left\langle\psi_{j} \mid \psi_{k}\right\rangle\right|^{2} L(E),
$$

where $A_{j k}=n_{j} p_{k}, n_{j}$, and $p_{k}$ are the populations of the conduction and valence subbands. The term $\left\langle\psi_{j} \mid \psi_{k}\right\rangle$ is the overlap integral of the envelope functions. The line shape function $L(E)$ is obtained joining a Lorentzian with a phenomenological broadening $\Gamma / 2=3.2 \mathrm{meV}$ on the low energy side and an exponential decay $\propto \exp \left(-E / k_{\mathrm{B}} T_{\mathrm{e}}^{j}\right)$ on the high energy side. For $P \geq 0.5 \mathrm{~W}$ an excellent reproduction of the PL is obtained considering the $j \rightarrow k$ transitions that have an overlap integral $>0.2$ and leaving $T_{\mathrm{e}}^{j}, A_{j k}$ as fitting parameters [8]. Despite the large value of the overlap integrals associated to the transitions $3 \rightarrow k$ and $2 \rightarrow k$ involving the lower laser level $(j=3)$ and the upper state of the phonon-assisted transition $(j=2)$, respectively, no evidence of PL bands associated to the above transitions is present in Fig. 1. This results demonstrates that the lower laser level $j=3$ is populated by a negligible fraction of carriers and that that electron-LO phonon interaction efficiently depletes the subband $j=2$.

The fitting parameters $T_{\mathrm{e}}^{j}$ are plotted in Fig. 2a, as a function of $P$ together with the measured $T_{\mathrm{L}}$ values. The small difference $(\approx 5 \mathrm{~K})$ between $T_{\mathrm{e}}^{1}$ and $T_{\mathrm{L}}$ at zero electrical power is a well known effect due to the heating of the electronic ensemble induced by the probe laser. We found that the electronic temperatures of the ground state subband $j=1$ increase linearly with $P$ with a slope $R_{\mathrm{e}}=$ $29.0 \mathrm{~K} / \mathrm{W}$, slightly larger than the thermal resistance $R_{\mathrm{L}}=\mathrm{d} T_{\mathrm{L}} / \mathrm{d} P=24.5 \mathrm{~K} / \mathrm{W}$. On the other hand, the temperature of the upper laser level $T_{\mathrm{e}}^{5}$ reaches $\approx 140 \mathrm{~K}$, 


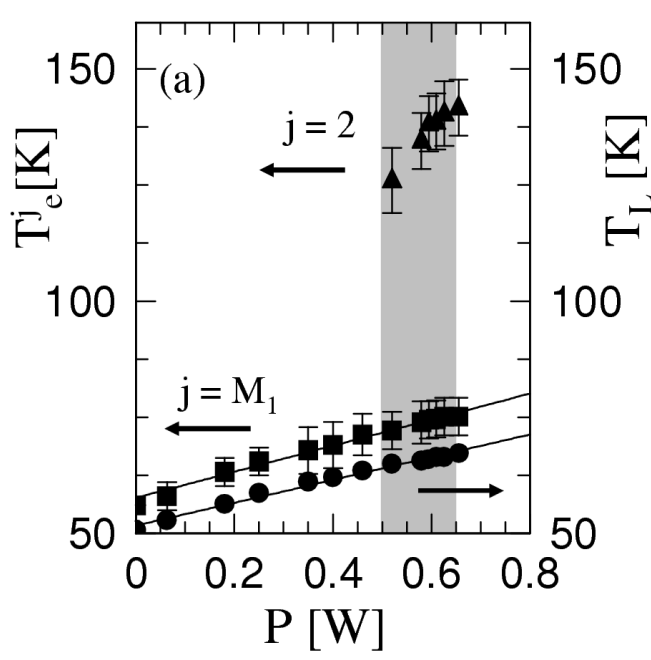

(c)

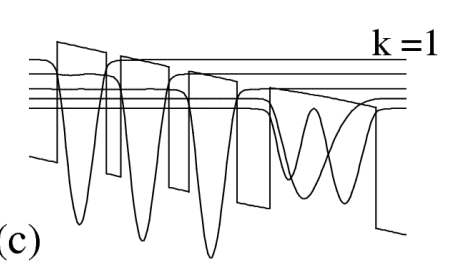

Fig. 2. (a) Mean lattice $T_{\mathrm{L}}(\bullet)$ and electronic temperatures $T_{\mathrm{e}}^{j} ; j=1(\boldsymbol{\bullet}) ; j=4$ in the active region of sample (a) measured as a function of the electrical power at a heat sink temperature $T_{\mathrm{H}}=50 \mathrm{~K}$. The lines are linear fits to the data. The shaded areas mark the lasing region. Conduction (b) and valence (c) band structures of sample (a) are calculated with a voltage drop of $46.5 \mathrm{mV}$ per stage using a self- consistent method based on the iterative solution of the Schrödinger and Poisson coupled equations. The layer thicknesses in $\mathrm{nm}$ (from left to right, starting at the injection barrier) are: $\underline{49} / 78 / \underline{23} / 76 / \underline{32} / 76 / \underline{52} / 168$. AlGaAs layers are shown in bold and the $52 \AA$ collector barrier is delta-doped at $n=2.25 \times 10^{10} \mathrm{~cm}^{-2}$ in the center. The energy levels are labeled using increasing integers starting from the ground state either in the conduction or valence bands.

i.e. it is higher by $\approx 80 \mathrm{~K}$ than $T_{\mathrm{L}}$. The existence of differences as high as $25-$ $40 \%$ in the subband electronic temperatures has been predicted by Monte Carlo simulations both in mid-IR and THz QCLs, [9] and already assessed experimentally in RP devices with a standard injector design [8]. One important implication of these findings is that the high $T_{\mathrm{e}}^{4}$ values lead to relatively fast non-radiative relaxation times $\tau_{4 \rightarrow 3,2} \approx 1.9 \mathrm{ps}$ that represents a key limiting factor for the high temperatures operation of this class of devices [8].

Figure 3a shows the results of the PL experiments obtained for sample (b). In the case of BTC devices, band-to-band transitions associated with the injector low energy miniband and upper laser level $j=2$ are visible, together with the heavyhole $(\mathrm{HH})$ and light-hole $(\mathrm{LH})$ exciton peaks arising from level 2, as expected from the calculation of the Debye screening length [8,9]. For the line shape analysis we used as fitting function a Lorentzian on the low energy side joined with an exponential decay on the high energy side for band-to-band transitions and a Lorentzian for the exciton peaks $[10,11]$. The experimental data have been 

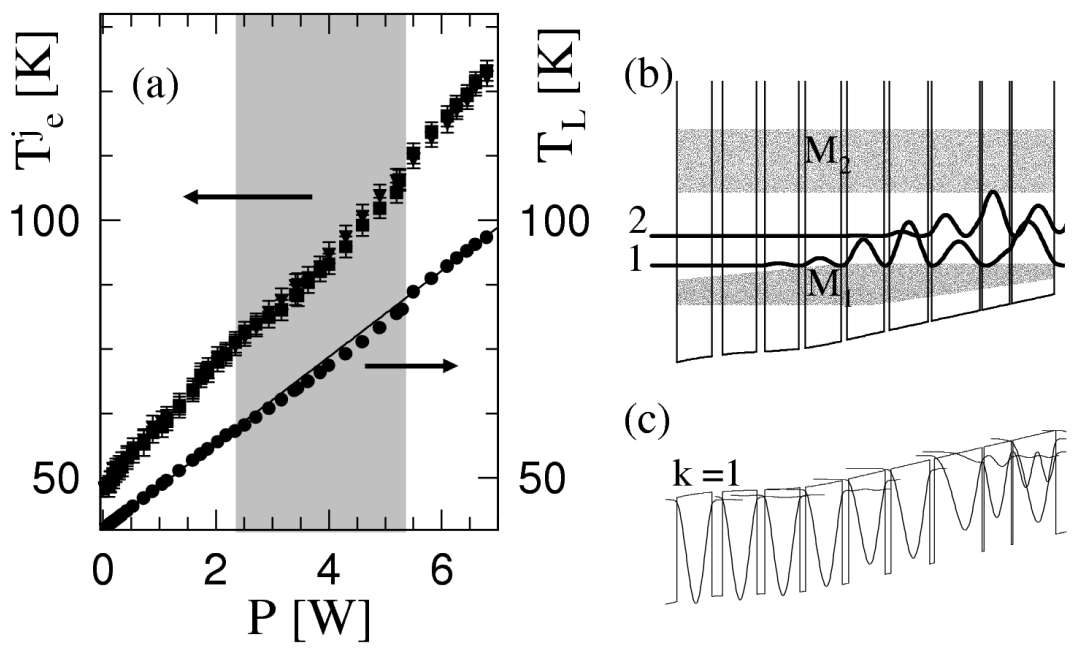

(c)

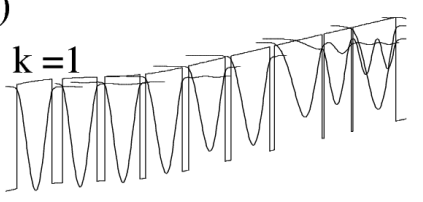

Fig. 3. Mean lattice temperature $T_{\mathrm{L}}(\bullet)$ and subband electronic temperatures $T_{\mathrm{e}}^{M_{1}}$ $(\mathbf{-}), T_{\mathrm{e}}^{2}(\boldsymbol{\nabla})$ in the active region of sample (b) measured as a function of the electrical power $P$ at a heat sink temperature of $45 \mathrm{~K}$. The lines are linear fits to the data. The shaded areas mark the lasing region. Conduction (b) and valence (c) band structures of sample (b) are calculated with a voltage drop of $27 \mathrm{mV}$ per stage using a self-consistent method based on the iterative solution of the Schrödinger and Poisson coupled equations. Starting from the injection barrier, the layer sequence of 1 period, in nanometers is (from right to left): $\mathbf{3 . 9} / 14.0 / \mathbf{0 . 6} / 9.1 / \mathbf{0 . 6} / 16.0 /$

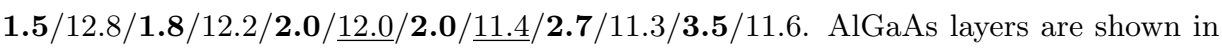
bold, and the underlined GaAs wells are $n$ doped at $1.6 \times 10^{16} \mathrm{~cm}^{-3}$. The shaded area $M_{1}$ represents the lowest energy miniband. $M_{2}$ marks the second miniband. The wave function square modulus of the upper and lower laser levels are labeled as 2 and 1, respectively. Valence subbands $k$ are labeled using increasing integers.

fitted leaving $T_{\mathrm{e}}^{j}$, the band intensities and the full width at half maximum of the $\mathrm{HH}$ and LH exciton peaks as fitting parameters. The electronic temperatures of subbands belonging to the lower injector minibands $M_{1}$ (see Fig. $3 \mathrm{~b}$ ) and to the upper state $j=2$ are nearly equal and increase linearly with $\mathrm{P}$ with a slope $R_{\mathrm{e}}=11.5 \mathrm{~K} / \mathrm{W}$, larger than the thermal resistance $R=\mathrm{d} T_{\mathrm{L}} / \mathrm{d} P=8.4 \mathrm{~K} / \mathrm{W}$. The existence of a common $T_{\mathrm{e}}$ value in the active region subbands demonstrates that the vast majority of electrons share the same electronic temperature, as already demonstrated in the case of BTC devices [11]. This is potentially beneficial for the extension of the device operation at higher temperatures. However, another parameter is expected to strongly influence the device temperature performance, i.e. the strength of the coupling between the electronic ensemble and the lattice. The latter is significantly influenced by the nature of the electron-lattice energy relaxation processes. To evaluate the efficiency of the above processes we extracted 
the electron-lattice coupling constant defined as: $\alpha=\Delta T / J$ where $\Delta T=T_{\mathrm{e}}^{j}-$ $T_{\mathrm{L}}, J$ is the current density and $j=1$ or $j=M_{1}$, in the case of RP or BTC devices, respectively. The latter assumption is due to the experimental evidence that the vast majority of electrons populate the ground state subbands. The lower the $\alpha$ constant, the higher the coupling between electrons and lattice [12]. We extracted the values $\alpha=30.2 \mathrm{Kcm} 2 / \mathrm{kA}$ and $\alpha=78.5 \mathrm{Kcm}^{2} / \mathrm{kA}$ for samples (a) and (b), respectively. In sample (a) the electron-lattice energy relaxation rate is controlled by the LO-phonon assisted transitions $3,2 \rightarrow 1$. The largest electronlattice coupling for sample (a) confirms the efficacy of its design that was aimed at increasing the matrix elements associated with the $3,4 \rightarrow 1,2$ transitions. Also, the energy separation between the above levels is slightly higher than $E_{\mathrm{LO}}$ at all values of the electric field above the alignment. The more efficient carrier thermalization in sample (a) reduces thermal backfilling of the subband $j=4$, helps in keeping the optical gain closer to the designed value, and thus improves optical performance. The higher $\alpha$ measured for sample (b) can be explained by considering that in BTC devices due to the low energy separation between most of subbands $(<0.036 \mathrm{eV})$ the electron-electron scattering becomes the dominant energy relaxation channel, differently from mid-IR or resonant-phonon RP THz QCL devices. Therefore, the inelastic electron-LO-phonon scattering, which couples the electron distribution to the lattice heat bath, does not allow cooling of electrons as efficiently as in devices that use the LO-phonon scattering as main depletion mechanism.

\section{TABLE}

Comparison between the electron-lattice coupling constants $\alpha$ of a set of investigated THz QCLs, classified following their emission frequency $(v)$, quantum design and type of waveguide ( $s p$ and $m-m$ indicate surfaceplasmon or double-metal waveguides, respectively).

\begin{tabular}{c|c|c|c|c}
\hline \hline Sample & $v[\mathrm{THz}]$ & Design & Waveguide & $\alpha\left[\mathrm{K} \mathrm{cm}^{2} / \mathrm{kA}\right]$ \\
\hline $\mathrm{a}$ & 1.9 & $\mathrm{rp}-\mathrm{sw}$ & $\mathrm{m}-\mathrm{m}$ & 30.2 \\
$\mathrm{~b}$ & 2.83 & $\mathrm{btc}$ & $\mathrm{sp}$ & 78.5 \\
$\mathrm{c}^{(13)}$ & 2.9 & $\mathrm{btc}$ & $\mathrm{sp}$ & 81.5 \\
$\mathrm{~d}^{(13)}$ & 2.9 & $\mathrm{btc}$ & $\mathrm{m}-\mathrm{m}$ & 82.5 \\
$\mathrm{e}^{(13)}$ & 2.9 & $\mathrm{btc}$ & $\mathrm{m}-\mathrm{m}$ & 82.2 \\
$\mathrm{f}^{(9)}$ & 2.5 & $\mathrm{btc}$ & $\mathrm{sp}$ & 81.0 \\
$\mathrm{~g}^{(11)}$ & 2 & $\mathrm{btc}$ & $\mathrm{sp}$ & 95.5 \\
$\mathrm{~h}^{(8)}$ & 3.2 & $\mathrm{rp}$ & $\mathrm{m}-\mathrm{m}$ & 42.0 \\
$\mathrm{i}^{(8)}$ & 2.8 & $\mathrm{rp}$ & $\mathrm{m}-\mathrm{m}$ & 24.0
\end{tabular}

These considerations are confirmed from the results, obtained in a set of previously investigated BTC and RP devices, reported in Table. In BTC samples 
the $\alpha$ values are always in the range $78-81 \mathrm{Kcm}^{2} / \mathrm{kA}$, except for sample $(\mathrm{g})$ where the further increase in $\alpha$ can be ascribed to the use of a lower conduction bandoffset and thus to an increase in the leakage channels. For RP and RP-SW devices $\alpha$ varies between $24 \mathrm{Kcm}^{2} / \mathrm{kA}$ and $42 \mathrm{Kcm}^{2} / \mathrm{kA}$. The $40 \%$ change in the coupling between electrons and lattice is due to the differences in the design of the phonon depletion schemes that influences the efficiency of LO-phonon assisted transitions. The above results demonstrate that a careful optimization of the inter- and intrasubband scattering via quantum design is thus necessary to assure a simultaneous better intra-subband coupling and a more efficient cooling of the electronic system with the lattice in order to extend $\mathrm{THz}$ quantum cascade laser operation in the temperature range of the thermoelectric coolers or higher.

\section{Acknowledgments}

The work was partly supported by Regione Puglia, PE-056. We are grateful to S. Kumar and Q. Hu for providing the selection of suitable RP devices and for helpful discussions.

\section{References}

[1] R. Köhler, A. Tredicucci, F. Beltram, H.E. Beere, E.H. Linfield, A.G. Davies, D.A. Ritche, R.C. Iotti, F. Rossi, Nature 417 156, 02 (20.)

[2] C. Walther, G. Scalari, J. Faist, H. Beere, and D. Ritchie, Appl. Phys. Lett. 89, 231121 (2006).

[3] V.B. Gorfinkel, S. Luryi, B. Gelmont, IEEE J. Quant. Electron. 32, 1995 (1996).

[4] S. Kumar, B. Williams, Q. Hu, J. Reno, Appl. Phys. Lett. 88, 121123 (2006).

[5] M.S. Vitiello, G. Scamarcio, V. Spagnolo, S.S. Dhillon, C. Sirtori, Appl. Phys. Lett. 90, 191115 (2007).

[6] M.S. Vitiello, G. Scamarcio, V. Spagnolo, J. Nanophoton. 1, 013514 (2007).

[7] V. Spagnolo, G. Scamarcio, H. Page, C. Sirtori, Appl. Phys. Lett. 84, 3690 (2004).

[8] M.S. Vitiello, G. Scamarcio, V. Spagnolo, B.S. Williams, S. Kumar, Q. Hu, J.L. Reno, Appl. Phys. Lett. 86, 11115 (2005).

[9] H. Callebaut, S. Kumar, B. S. Williams, Q. Hu, J.L. Reno, Appl. Phys. Lett. 83, 207 (2003).

[10] M.S. Vitiello, G. Scamarcio, V. Spagnolo, L. Mahler, T. Losco, A. Tredicucci, H.E. Beere, D.A. Ritchie, Appl. Phys. Lett. 88, 241109 (2006).

[11] M.S. Vitiello, G. Scamarcio, V. Spagnolo, C. Worral, H.E. Beere, D.A. Ritchie, C. Sirtori, J. Alton, S. Barbieri, Appl. Phys. Lett. 89, 131114 (2006).

[12] M.S. Vitiello, G. Scamarcio, V. Spagnolo, A. Lops, Q.Yang, C. Manz, W. Bronner, K. Köhler, J. Wagner, Appl. Phys. Lett. 90, 121109 (2007).

[13] M.S. Vitiello, G. Scamarcio, V. Spagnolo, J. Alton, S. Barbieri, C. Worral, H.E. Beere, D.A. Ritchie, C. Sirtori, Appl. Phys. Lett. 89, 021111 (2006). 